\title{
Valoração econômica dos benefícios ambientais percebidos pela população da bacia do Educandos provenientes do PROSAMIM
}

\author{
Kellem Andrezza Araújo FREITAS ${ }^{1}$, José Barbosa FILHO ${ }^{2}$, Nabor Silveira PIO ${ }^{3}$, Frederico Fonseca SILVA \\ Larice Silva MORAES 5
}

\begin{abstract}
RESUMO
A poluição ambiental observada ao longo de anos nos igarapés de Manaus (Amazonas-Brasil), associado ao processo de degradaçáo social da população que vive nessas áreas foi o que motivou a concepçáo do Programa Social e Ambiental dos Igarapés de Manaus - PROSAMIM. O Programa busca a recuperação ambiental dos igarapés e a melhoria social das famílias que vivem em situaçáo de risco nas margens. O objetivo principal da pesquisa consistiu em avaliar economicamente os benefícios ambientais percebidos pela população da bacia do Educandos provenientes do PROSAMIM, usando o Método de Valoraçáo Contingente - MVC para estimar a disposiçáo a pagar das pessoas pela melhoria ambiental obtida. Foi estimado um tamanho de amostra correspondente a 1.070 questionários, levando em conta um erro de 3\% e um nível de significância de 5\%. Concluiu-se que a disposição a pagar mensal das pessoas é $\mathrm{R} \$ 13,73$ e o valor econômico total para a melhoria ambiental relativa à execução do PROSAMIM é R\$ 46.325.074,92 por ano. Analisando a influência de variáveis socioeconômicas na probabilidade de aceitar pagar por benefícios ambientais foi observado que somente o nível de renda apresenta significância estatística e o coeficiente angular positivo da variável, indica que a probabilidade de aceitar pagar o valor sugerido é maior em pessoas que têm o nível de renda mais alto. Finalmente, pode ser concluído que as açóes do PROSAMIM têm gerado melhorias significantes para a populaçáo da cidade de Manaus, tanto do ponto de vista social quanto ambiental.
\end{abstract}

PALAVRAS-CHAVE: Bacia hidrográfica; Economia ambiental; Método de valoração contingente.

\section{Economic valuation of environmental benefits perceived by the Educandos basin population proceeding from PROSAMIM}

\begin{abstract}
The environmental pollution observed down through the years in the streams of Manaus, linked to the process of social degradation on the populations which live in these areas was the motive for conceiving PROSAMIM - Programa Social e Ambiental dos Igarapés de Manaus. The Program seeks the environmental recuperation of streams and the social improvement of families that live a risk situation on the stream banks. The main target of this research consists in the economical evaluation of environmental benefits perceived by the population in the Educandos basin proceeding from PROSAMIM, using the Contingent Valuation Method - CVM to estimate individual willingness to pay for the environmental improvement. We estimated a sample size corresponding to 1,070 questionnaires, taking into account an error of 3\% and significance level of $5 \%$. In accordance with the results obtained, we concluded that the people were willing to pay R $\$ 13.73$ per month toward implementing the PROSAMIM, for an annual total of R $\$ 46,325,074.92$ per year. Analyzing the influence of socioeconomic variables on the acceptance probability of paying for environmental benefits, we concluded that the probability was greater among those with a higher income. We also concluded that, the actions of PROSAMIM have generated significant improvements for the population of Manaus from the environmental as well as the social point of view.
\end{abstract}

KEYWORDS: Hidrografic basin; Environmental economics; Contingent valuation method.

\footnotetext{
1 Universidade Federal do Amazonas - UFAM, E-mail: kfreitas@ufam.edu.br

2 Universidade Federal do Amazonas - UFAM, E-mail: jbarbosa@ufam.edu.br

${ }^{3}$ Universidade Federal do Amazonas - UFAM, E-mail:nspio@ufam.edu.br

${ }^{4}$ Universidade Federal de Roraima - UFAM, E-mail: fredfonseca@rogers.com

${ }^{5}$ Universidade Federal do Amazonas - UFAM, E-mail: larice_moraes@hotmail.com
} 


\section{INTRODUÇÃO}

A variedade de produtos ofertados pelo mercado e os padróes de consumo ditados pela sociedade capitalista têm gerado sérias conseqüências à conservaçáo dos recursos naturais e a qualidade de vida da população. Assim, o que antes era considerado apenas sob o ponto de vista social, passou a ter nova dimensão devido ao aumento das ameaças globais como as mudanças climáticas, o desmatamento e a extinção de espécies vegetais e animais, tornando-se necessário analisar as questóes ambientais também sob a ótica econômica.

A interaçáo entre o meio ambiente e o sistema econômico deve-se aos impactos que a economia provoca no ambiente e vice-versa. Assim, conhecer o valor dos recursos naturais e incluir esses valores na análise econômica é uma tentativa de corrigir as tendências negativas do mercado.

Para Marques e Comune (1997), ao atribuir preço aos recursos naturais, a economia ambiental analisa os custos e os benefícios alocados ao meio ambiente, conferindo-lhe significado que vai além da teoria de mercado, no qual se encontram incorporadas atribuiçóes ecológicas e sociais.

Deste modo, a disposição a pagar pela conservação de um determinado recurso natural reflete as preferências individuais por bens ambientais. $\mathrm{O}$ valor econômico de um ativo ambiental é caracterizado como uma medida de preferência. $\mathrm{O}$ resultado da valoração é representado em termos monetários e a utilização desta medida possibilita uma comparação entre o valor do meio ambiente e o valor de um projeto de desenvolvimento, permitindo uma análise do custo de oportunidade socioambiental de um projeto (PEARCE e TURNER, 1985).

A cidade de Manaus possui características geográficas peculiares, como por exemplo, a existência de cursos d'água em toda sua extensão urbana, o que poderia lhe conferir posição de destaque em termos de infra-estrutura e beleza cênica. No entanto, a existência dos igarapés na área urbana da cidade tem apresentado sérios problemas pela falta de conservação dos recursos hídricos, degradados pela ocupação humana ao longo de suas margens e pela poluição doméstica e industrial em suas águas. Cabe ao poder público a tarefa de empreender esforços na tentativa de mitigar estes danos ambientais.

Neste sentido, o Programa Social e Ambiental dos Igarapés de Manaus - PROSAMIM surgiu com a proposta de prover a recuperaçấo ambiental dos igarapés, bem como a melhoria social das famílias que residem nas áreas de influência direta e indireta dos igarapés.

O Programa, um mecanismo de política pública que tem buscado a revitalizaçáo dos igarapés, representa neste estudo o parâmetro de avaliaçáo das mudanças ambientais geradas, possibilitando identificar como tais alteraçóes são percebidas pela população local.
Portanto, a pesquisa foi realizada pela necessidade de calcular, com base nas preferências humanas, um valor econômico que represente a importância ambiental dos igarapés da bacia do Educandos para a cidade de Manaus, por meio da utilizaçáo do método de valoração contingente que busca estimar a disposiçáo dos indivíduos de pagar pela melhoria dos igarapés.

O estudo visa, desta forma, contribuir para a criação e manutenção de políticas públicas socioambientais na cidade à medida que a incorporaçấo das externalidades ambientais na análise de projetos agregue valor aos recursos naturais, permitindo inserir o meio ambiente nas estratégias de desenvolvimento econômico local. Além disso, o valor ambiental dos igarapés, determinado a partir da disposição a pagar da população expressa a preferência dos indivíduos sobre as alteraçôes em seu meio ambiente e com isso, os anseios por uma melhor qualidade de vida.

\section{MATERIAL E MÉTODOS}

O procedimento específico de valoração ambiental adotado neste estudo foi o Método de Valoraçáo Contingente - MVC que se baseia na percepção ambiental dos indivíduos, de modo a captar a disposição a pagar (DAP) ou a disposição a receber (DAR) pela conservação ou permitir a degradaçáo, respectivamente, de um determinado recurso ambiental. No caso desta pesquisa, foi obtida a disposiçấo a pagar dos indivíduos para a manutenção dos benefícios advindos do processo de revitalização dos igarapés da bacia do Educandos.

Destaca-se que este método foi desenvolvido por Robert Davis em 1963 em sua tese de doutoramento na Universidade de Harvard. Nesta pesquisa, o autor procurou simular o comportamento do mercado, buscando captar a máxima disposição a pagar dos pesquisados por uma área recreacional na floresta de Maine, nos Estados Unidos, oferecendo sucessivos valores até que o entrevistado aceitasse ou rejeitasse a proposta de pagamento do recurso natural (SILVA, 2003).

No caso desta pesquisa, foi obtida a disposição a pagar dos indivíduos para a manutenção dos benefícios advindos do processo de revitalizaçấo dos igarapés da bacia do Educandos.

De acordo com Ortiz (2003), a grande vantagem na utilizaçáo do método de valoração contingente sobre os demais métodos de valoração econômica ambiental é que esta metodologia é a única que permite a estimação de valores de existência, tendo em vista que a obtenção das preferências dos pesquisados sobre determinado recurso natural pode ser realizada em um cenário que está ou não relacionado ao uso prévio do recurso natural pelos indivíduos.

Barbosa Filho (2005) destaca que o MVC agrega um conjunto de técnicas de pesquisa utilizadas para estimar o valor econômico de bens e serviços ambientais com base na 
preferência dos consumidores. A metodologia é considerada de Provisão Local (Local Provisional), pois visa solucionar um problema peculiar de certo campo de interesse. Com isso, a aplicabilidade do método nesta pesquisa é justificada pelo fato de elucidar um problema específico na cidade de Manaus que consiste em mensurar um valor econômico que represente a importância ambiental dos igarapés da bacia do Educandos.

A população investigada foi restrita aos residentes na cidade de Manaus por no mínimo cinco anos, maiores de 18 anos e que possuem renda. Para tanto, foi estimado um tamanho de amostra correspondente a 1.070 pesquisados, levando em conta um erro de $3 \%$ e um nível de significância de $5 \%$.

A fim de captar a percepção da população pela melhoria ambiental de forma mais representativa e menos tendenciosa, o instrumento de coleta de dados, no caso o questionário estruturado, foi distribuído aleatoriamente entre as seis zonas urbanas da cidade de Manaus e náo somente entre os moradores do entorno dos igarapés da bacia do Educandos.

O questionário foi baseado nos estudos empíricos citados por May e Pessoa (1996). Contém uma pequena nota explicativa sobre o objetivo do instrumento de coleta, bem como perguntas relacionadas a idade, ao sexo, ao setor de trabalho, a renda familiar, a escolaridade e a naturalidade do pesquisado, com o objetivo de identificar a influência dessas variáveis para a determinação do valor ambiental dos igarapés em questão. Além disso, o questionário contempla a descrição da mudança ambiental gerada pelo PROSAMIM e os aspectos a serem avaliados, sejam eles positivos ou negativos.

A área de estudo foi a bacia hidrográfica do Educandos, composta pelos seguintes igarapés: Bittencourt, Manaus, Mestre Chico, Cachoeirinha e Quarenta. Possui $44.87 \mathrm{~km}^{2}$ localizando-se na porção sudeste de Manaus.

Geograficamente, a bacia do Educandos é constituída em um polígono de orientação N-NE que tem como limites as latitudes $3^{\circ} 4^{\prime} 00^{\prime \prime} \mathrm{S}$ e $3^{\circ} 9^{\prime} 00^{\prime \prime} \mathrm{S}$ e as longitudes $60^{\circ} 1^{\prime} 30^{\prime \prime} \mathrm{W}$ e $59^{\circ} 55^{\prime} 30^{\prime \prime} \mathrm{W}$.

Suas nascentes estão localizadas no Refúgio de Vida Silvestre Sauim-Castanheiras, no Distrito Industrial e em terrenos dos bairros Zumbi dos Palmares e Armando Mendes. Os canais de seus principais igarapés drenam para o Rio Negro com direção predominante N-NE (PROSAMIM, 2004).

\section{RESULTADOS E DISCUSSÃO}

Foram pesquisados 1.070 indivíduos residentes na cidade de Manaus. Deste total, apenas 200, correspondentes a 19\%, mostraram-se dispostos a pagar para a melhoria ambiental dos igarapés da bacia do Educandos, enquanto 870 pesquisados, correspondentes a $81 \%$ não aceitaram contribuir com nenhum dos valores propostos para a manutençáo dos benefícios ambientais provenientes do PROSAMIM, conforme Figura 1.

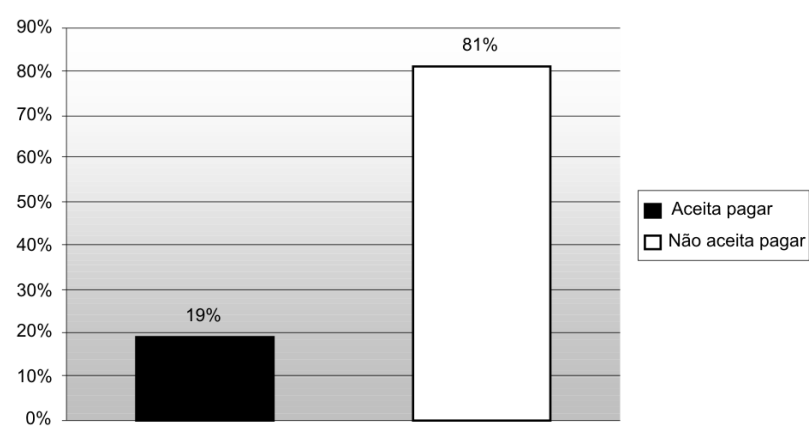

Figura 1 - Disposição a pagar dos pesquisados para a melhoria ambiental dos igarapés da bacia do Educandos, Manaus/AM. Fonte: Pesquisa (2008).

Os 870 entrevistados que não estáo dispostos a pagar para a melhoria ambiental dos igarapés da bacia do Educandos apresentaram diversos motivos para justificar a sua decisão. A Tabela 1 apresenta as justificativas apontadas.

Com base nas justificativas apresentadas, percebe-se que $58 \%$ dos motivos mais citados demonstram o descrédito na classe governante pela administraçấo dos recursos públicos e atitudes de protesto ao responsabilizar o governo pelos problemas ambientais.

Neste contexto, pode-se inferir que os motivos apontados pelos indivíduos para náo aceitar os valores propostos para a conservaçáo dos igarapés refletem a desconfiança dos manauaras na classe governante. Além disso, a atual conjuntura econômica, política e social do Brasil não prioriza os projetos de educação ambiental para a valorizaçáo e conservação do meio ambiente e o baixo nível de renda da maioria dos brasileiros ainda é um fator condicionante, que limita as pessoas a praticarem açôes que contribuam para o bem-estar coletivo.

Os resultados apresentados sobre o perfil dos pesquisados e a sua disposição a pagar pelos benefícios ambientais nos igarapés da bacia do Educandos revelam que as preferências dos indivíduos pela qualidade ambiental do recurso valorado estáo condicionadas ao contexto social e econômico no qual se encontram inseridos. Assim, o fato da maioria dos entrevistados náo aceitar os valores estipulados, náo significa que estes não almejem por melhorias ambientais nos igarapés ou não valorizem sua função ecossistêmica para a conservação da biodiversidade existente no local.

Percebe-se que os dados revelados nesta pesquisa são similares aos estudos de Mattos (2006), que apresenta um percentual de $55 \%$ de indivíduos que também não aceitam pagar nenhum valor para a conservação das áreas 
Tabela 1 - Justificativas apresentadas pelos pesquisados para não aceitar os valores propostos para a melhoria ambiental dos igarapés da bacia do Educandos, Manaus/AM. Fonte: Pesquisa (2008).

\begin{tabular}{lccc}
\hline Item & Justificativas & N. de vezes citada & \% de vezes citada \\
\hline 1 & Não confia que os valores arrecadados sejam utilizados para a melhoria dos & 273 & 30 \\
\hline 2 & igarapés & 243 & 28 \\
3 & É de responsabilidade do governo cuidar do meio ambiente & 121 & 14 \\
\hline 4 & A renda não permite & 69 & 8 \\
\hline 5 & Já paga muitos impostos e taxas & 52 & 6 \\
\hline 6 & Já contribui com instituições que cuidam do meio ambiente & 40 & 5 \\
\hline 7 & Deve haver atividade de educação ambiental e mutirão para limpeza e & 33 & 4 \\
\hline 8 & conservação dos igarapés & 22 & 3 \\
\hline 9 & Não tem interesse sobre o assunto & 12 & 1 \\
\hline 10 & Outros motivos & 5 & 1 \\
\hline
\end{tabular}

de preservação permanentes da microbacia do Ribeirão São Bartolomeu em Viçosa (MG). Resultado semelhante foi obtido por Brugnaro (2000), onde 58\% dos pesquisados náo estavam dispostos a pagar para a recuperaçáo da mata ciliar da bacia do Rio Corumbataí (SP).

Provavelmente, o número de brasileiros dispostos a pagar alguma quantia pela conservação das bacias hidrográficas é menor, se comparado à disposição a pagar por outros recursos naturais que utilizam diretamente, como é o caso de praias e parques. Na pesquisa de Martins (2000) apud Mattos (2006), 75,8\% dos entrevistados estavam dispostos a pagar pelos recursos ambientais da praia de Jericoacoara (CE). Resultado equivalente foi obtido nos estudos de Silva (2003), que encontrou um índice de $68 \%$ de disposição a pagar para a conservação do Parque Ambiental Chico Mendes no Acre.

\section{DISPOSIÇÃO A PAGAR DA POPULAÇÃO PARA A MELHORIA AMBIENTAL DOS IGARAPÉS DA BACIA DO EDUCANDOS}

Considerando os $19 \%$ dos pesquisados que afirmaram estar dispostos a pagar alguma quantia para a melhoria ambiental dos igarapés da bacia do Educandos foi obtida a tabela de regressão logística que estabelece a correlação entre a variável independente, representada pela disposição a pagar e a variável dependente, que se refere a probabilidade do indivíduo aceitar o valor estipulado (Tabela 2).

Para estimar a disposiçáo a pagar mensal, anual e o valor econômico total para a manutenção dos benefícios ambientais provenientes do PROSAMIM, foi realizada a seguinte equação de regressão.

$$
\mathrm{Pi}=1 /\left[1+\mathrm{e}^{-(1,2837-0,093482 \mathrm{DAP})}\right] \quad(\mathrm{p}=0,000000) ;
$$

Onde Pi refere-se a probabilidade de aceitação dos valores propostos pelos indivíduos.

Disposição a pagar mensal $=-(1,2837 /-0,093482)=$ R\$ 13,73 /família /mês

Disposição a pagar anual $=13,73 \times 12=\mathrm{R} \$ 164,76 /$ família /ano

Disposição a pagar total $=164,76 \times 281,167$ (número de famílias na cidade de Manaus) $=\mathrm{R} \$ 46.325 .074,92$ /ano

Com base nesta equação, o valor econômico total obtido foi de $\mathrm{R} \$ 46.325 .074,92$ por ano para a melhoria ambiental dos igarapés da bacia do Educandos, representando os benefícios ambientais associados à implementaçáo das açóes do PROSAMIM na cidade de Manaus.

A partir da regressão logística, proposta pelo método de valoração contingente foi possível estabelecer a curva de probabilidade de aceitação acumulada, representada pela Figura 2, na qual apresenta um nível de significância estatística alto, o que vem a confirmar sua aplicabilidade.

A curva de probabilidade de aceitaçáo acumulada indica que aproximadamente $60 \%$ dos indivíduos estão dispostos a pagar a quantia de $\mathrm{R} \$ 13,73$ para a melhoria ambiental dos

Tabela 2 - Tabela de regressão logística que estabelece a correlação entre as variáveis dependentes e independentes para estimar a disposição a pagar para a melhoria ambiental dos igarapés da bacia do Educandos, Manaus/AM. Fonte: Pesquisa (2008).

\begin{tabular}{lcccc}
\hline Estimativa & Coeficiente & Desvio Padrão & Significância & Probabilidade \\
\hline Constante & 1,2837 & 0,1755 & 7,31 & 0,000 \\
Disposição a Pagar & $-0,093482$ & 0,007304 & $-12,80$ & 0,000 \\
\hline
\end{tabular}




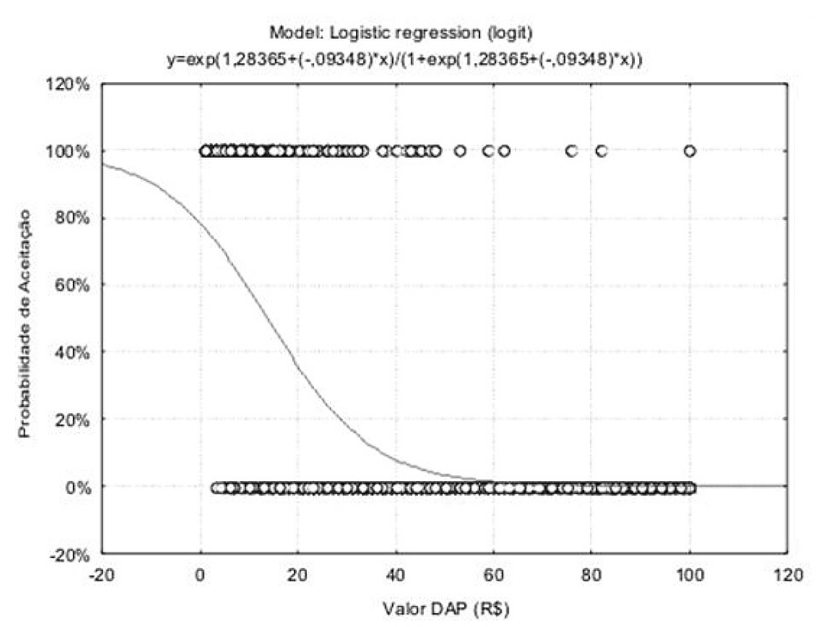

Figura 2 - Curva de probabilidade de aceitação acumulada dos valores propostos aos pesquisados. Fonte: Pesquisa (2008).

igarapés. Além disso, a probabilidade de aceitação dos valores propostos diminui quanto maior forem estes valores.

\section{INFLUÊNCIA DAS VARIÁVEIS SOCIOECONÔMICAS DOS INDIVÍDUOS PARA A DETERMINAÇÃO DO VALOR ECONÔMICO DOS IGARAPÉS}

As tomadas de decisóes relacionadas ao meio ambiente envolvem uma série de aspectos vinculados com princípios morais, culturais, religiosos e condiçôes socioeconômicas que transcendem o campo das opçóes específicas de consumo e estariam mais próximas das questôes filosóficas sobre a relação do homem com a natureza.

Com isso, foi analisada a influência das variáveis socioeconômicas dos pesquisados sobre a probabilidade de aceitação dos montantes estipulados na disposição a pagar por meio de um modelo de regressão múltipla com variáveis qualitativas (Tabela 3).

De acordo com os resultados apresentados, foi observado que a regressão logística como um todo é significante estatisticamente e a interpretaçáo do sinal associado ao coeficiente angular dessas variáveis vem de encontro ao que propóe a teoria econômica, ou seja: o sinal negativo associado a variável disposição a pagar indica que a probabilidade de aceitação do montante estipulado ao respondente diminui de acordo com o aumento dos valores propostos.

Neste sentido, foi identificado por meio da significância estatística para um $\alpha=0,05$ que as únicas variáveis socioeconômicas que apresentam relaçáo com a probabilidade de aceitação de um valor estipulado são a disposição a pagar e o nível de renda.

Para a variável renda, o sinal positivo do coeficiente angular indica que os indivíduos com maior nível de renda possuem maior probabilidade de aceitar pagar pelos valores propostos. Para Pearce \& Turner (1985), os indivíduos com maior poder aquisitivo demandam melhorias ambientais por já terem satisfeito as suas necessidades básicas e materiais. Para tanto, almejam melhorar sua qualidade de vida e, conseqüentemente, seu nível de bem-estar, o que muitas vezes é alcançado por meio das melhorias ambientais.

Este resultado também está em consonância com Mota (2006) que afirma que o indivíduo é um ser racional no processo de escolha, maximizando sua satisfaçáo a partir do preço do recurso natural e de sua restriçáo orçamentária.

O fato das demais variáveis socioeconômicas (sexo, idade, escolaridade e setor de trabalho) nâo apresentarem relaçâo com a probabilidade de aceitação de um valor estipulado para a melhoria ambiental da bacia do Educandos, deve-se ao fato de ter ocorrido uma tendência não proposital na amostra levantada, uma vez que $71 \%$ dos indivíduos pesquisados são jovens e $74 \%$ da amostra não possui nível superior.

Além disso, devido a maioria dos pesquisados não aceitar os valores estipulados, não significa que estes não almejem por melhorias ambientais nos igarapés ou não valorizem sua função ecossistêmica para a conservação da biodiversidade existente no local. Ao contrário, a restrição orçamentária

Tabela 3 - Modelo de regressão múltipla com variáveis qualitativas para estimar a correlação das variáveis qualitativas com a probabilidade do pesquisado aceitar os valores propostos para a recuperação ambiental dos igarapés da bacia do Educandos, Manaus/AM. Fonte: Pesquisa (2008).

\begin{tabular}{|c|c|c|c|c|}
\hline Variáveis & Coeficiente & Desvio Padrão & Significância & Probabilidade \\
\hline Constante & 0,4515 & 0,6184 & 0,73 & 0,465 \\
\hline Disposição a Pagar & $-0,12488$ & 0,01025 & $-12,18$ & 0,000 \\
\hline Idade & 0,00022 & 0,01065 & 0,02 & 0,984 \\
\hline Sexo & 0,0061 & 0,2359 & 0,03 & 0,979 \\
\hline Trabalho & $-0,2577$ & 0,2513 & $-1,03$ & 0,305 \\
\hline Renda & 0,00071919 & 0,00008088 & 8,89 & 0,000 \\
\hline Escola 1 & 0,3527 & 0,4214 & 0,84 & 0,403 \\
\hline Escola 2 & 0,1425 & 0,3791 & 0,38 & 0,707 \\
\hline Naturalidade & $-0,1183$ & 0,2431 & $-0,49$ & 0,626 \\
\hline
\end{tabular}

* 0 s valores em vermelho representam as variáveis com significância estatística para um a $=0,05$ 
da maioria dos entrevistados é um fator condicionante que limita os indivíduos a praticar ações que contribuam para o bem-estar coletivo.

São recomendaçôes propostas por esta pesquisa que os valores estimados para a melhoria ambiental dos igarapés da bacia do Educandos sirvam de parâmetros para justificar o aporte financeiro em projetos que visem à manutenção das funçôes socioambientais do recurso natural. Além disso, são esperadas que as externalidades relacionadas às açóes do PROSAMIM sejam internalizadas pelo mercado, agregando valor aos igarapés e resultando em benefícios ambientais para toda a população manauense.

\section{CONCLUSÕES}

Conclui-se que o presente estudo levou ao conhecimento da comunidade científica, o valor de existência dos igarapés da bacia do Educandos, por meio de uma metodologia que captou, de forma direta, a percepçáo da população sobre as melhorias ambientais provenientes do PROSAMIM, buscando subsidiar futuras pesquisas e contribuir para a criação de políticas públicas ambientais com foco na conservação dos igarapés da cidade de Manaus.

A disposiçấo a pagar da população para a revitalização dos igarapés da cidade de Manaus reflete o nível de bem-estar dos indivíduos relacionado a conservação e a manutenção deste recurso natural, condicionado ao contexto social e econômico no qual encontram-se inseridos. Além disso, os resultados desta pesquisa revelam um possível mercado para esse recurso ambiental e refletem como seria o comportamento dos manauenses, caso ele existisse.

\section{AGRADECIMENTOS}

Agradecemos à CAPES pela concessão de bolsa de pesquisa, possibilitando a viabilização deste estudo.

\section{BIBLIOGRAFIA CITADA}

Barbosa Filho, José. 2005. Valuation model for environmental analysis of proposed investment. Tese de Doutorado em Engenharia de Produção, Universidade Federal de Santa Catarina, Florianópolis, Santa Catarina. 199pp. (in Portuguese)
Brugnaro, Caetano. 2000. Value assigned by the riparian population of the Corumbatai river, São Paulo, Brazil. Tese de Doutorado em Economia Aplicada, Escola Superior de Agricultura Luiz de Queiroz, Universidade de São Paulo, Piracicaba, São Paulo. 146pp. (in Portuguese)

Marques, João Fernando; Comune, Antônio Evaldo. 1997. The neoclassical theory and the environmental valuation.In: Romeiro, Ademar Ribeiro, et al. Environmental Economics: Theory, policy and management of regional spaces. Campinas: UNICAMP. p.21-42. (in Portuguese)

Mattos, Ana Dantas Mendez de. 2006. Environmental valuation of permanent preservation areas of the Sao Bartolomeu creek, in Viçosa, Brazil. Dissertação de Mestrado em Ciência Florestal, Universidade Federal de Viçosa, Minas Gerais. 91pp. (in Portuguese)

May, P. H. 1996. Ecological economics: applications in Brazil. Rio de Janeiro: Campus. 218pp. (in Portuguese)

Mota, José Aroudo. 2006. The value of nature: economics and politics of natural resources. Rio de Janeiro: Garamond. 198pp. (in Portuguese)

Ortiz, Ramon Arigoni. 2003. Economic valuation of environmental. In: May, Peter H; Lustosa, Maria Cecília; Vinha, Valéria da. Environmental Economics: theory and practice. 5.ed. Rio de Janeiro: Campus. p.81-99. (in Portuguese)

Pearce, David W; Turner, R Kerry. 1985. Environmental Economics. México: Fondo de Cultura Económica. 378pp. (in Spanish)

Pessoa. R.E.C. 1996. The contingent valuation method: an attempt to valuation of environmental assets of Roraima, Brazil. Dissertação de Mestrado em Economia, Universidade Federal de Pernambuco, Recife. 91pp. (in Portuguese)

Silva, Rubicleis Gomes da. 2003. Valuation of the environmental park Chico Mendes, Rio Branco, Brazil: application of probabilistic method referendum with bidding games. Dissertação de Mestrado em Economia Aplicada, Universidade Federal de Viçosa, Minas Gerais. 125pp. (in Portuguese)

Social and Environmental Program of the streams of Manaus, Brazil. 2004. Relatório de impacto ambiental-RIMA. Manaus. 140pp. (in Portuguese)

Recebido em 18/12/2008

Aceito em 27/11/2009 